\title{
IMPURITIES REMOVAL PROCESS FROM THE VACUUM INDUCTION FURNACE CHARGE: A NUMERICAL STUDY
}

\author{
PIOTR BULIŃSKI ${ }^{1}$, JACEK SMOLKA ${ }^{1}$, SLAWOMIR GOLAK ${ }^{2}$, ROMAN PRZYLUCKI $^{2}$, \\ MICHAL PALACZ ${ }^{1}$, GRZEGORZ SIWIEC ${ }^{3}$, JAKUB LIPART $^{3}$ \& LESZEK BLACHA ${ }^{3}$ \\ ${ }^{1}$ Institute of Thermal Technology, Silesian University of Technology, Poland \\ ${ }^{2}$ Department of Industrial Informatics, Silesian University of Technology, Poland \\ ${ }^{3}$ Institute of Metal Technology, Silesian University of Technology, Poland
}

\begin{abstract}
The technology of mental melting in a vacuum induction furnace enables the efficient removal of impurities and provides an opportunity to melt refractory metals, such as titanium. These materials can be applied in cutting edge technologies, such as aviation (turbine blades) and biotechnology (prosthesis and implants). To control metallurgical heat and mass processes within an induction furnace, measurements and a numerical analysis can be conducted. In this paper, numerical approaches are discussed. Simulation requires the development of vacuum induction furnace coupling between fluid dynamics and electromagnetic fields. The proposed numerical domain was modelled as a threedimensional slice with a properly defined periodic boundary condition. To define the analysed electromagnetic problem, a set of Maxwell differential equations was specified. A fluid dynamics sub-model was composed of the mass and momentum conservation equations using the volume of fluid multiphase formulation, two-equation $\mathrm{k}-\varepsilon$ turbulence model and species transport to track the inclusion position within the melt. The main purpose of this study was an examination of the impurities removal process via the free surface of the melt within an induction furnace. The coupled computations were performed for five operating conditions, including different power inputs of the inductor. The results indicated a strong influence of the inductor power on the free surface area and therefore on the purification process intensity.

Keywords: purification process, inclusion removal, vacuum induction furnace, multiphase flow, coupling procedure, computational fluid dynamics.
\end{abstract}

\section{INTRODUCTION}

Induction furnaces with cold crucibles are modern melting devices that are aimed at producing pure metals and alloys of the highest purity. Their unique feature is the ability to remove both volatile impurities and gases dissolved in the melt. The units are fitted with a crucible that consists of electrically insulated and water-cooled segments composed of copper. The crucible is placed in a cylindrical inductor that serves as a source of electromagnetic field. The advantage of this technology is the limited contact of the metal with the crucible. The side surfaces of the melt are repelled by the electrodynamic forces from the crucible.

To control the metallurgical heat and mass processes within an induction furnace, measurements and a numerical analysis can be performed. The literature review indicates that the experimental portion of the research is more advanced than its modelling counterpart. Numerical computations of induction furnaces are primarily focused on three groups of problems: free surface flow [1], induction heating [2] and an impurities removal process [3]. To successfully describe processes inside the furnace, the development of two-way coupling between an electromagnetic sub-model and a fluid dynamics sub-model.

In previous numerical studies of the research team [4], this coupling procedure was developed and examined for different operating conditions and material properties [5]. The 
last investigation [6], which was the most comprehensive analysis, indicated that the proposed mathematical model and coupling procedure yields reasonable accuracy in terms of the heat transfer and free surface prediction despite its simplicity.

The main objective of this study was the numerical investigation of the free surface and impurities removal process within an induction furnace. The considered charge was alloy of copper and lead with an initial $2 \%$ of the lead mass fraction. The coupled computations were performed for five operating conditions, including different power inputs of the inductor. The results indicated a strong influence of the inductor power on the free surface area and therefore on the purification process intensity.

\section{MATHEMATICAL MODEL}

The mathematical model was formulated for the real vacuum induction furnace produced by Seco-Warwick and located at the Institute of Metal Technology of the Silesian University of Technology in Katowice, Poland. To attain a high vacuum, this unit is equipped with a system of three vacuum pumps. This system provides a minimal pressure of $10 \mathrm{mPa}$. The maximum inductor power for this unit is of $75 \mathrm{~kW}$.

The geometrical model in Fig. 1 was comprised a small part of the vacuum chamber. The electromagnetic sub-model was denoted by a green line, whereas a red line corresponds to the boundaries of the fluid dynamics sub-model. To simplify the mathematical description of the electromagnetic sub-model, a tangential direction was negligible, and the numerical domain had two-dimensional axisymmetric geometry. The fluid dynamics geometry consisted of two crucibles (inner and outer): molten charge (copper-lead alloy) and the surrounding air. For the electromagnetic sub-model, the copper inductor was introduced. To properly simulate the magnetic field, the surrounding air was extended compared with the fluid dynamics. Once the geometry was developed, the next step was numerical discretisation. The electromagnetic mesh was small, and triangular elements were primarily generated. The fluid dynamics sub-model grid consisted of 100,000 quadrilateral elements. The electromagnetic sub-model had a small grid with 25,000 final elements. Both grids are presented in Fig. 1.

The electromagnetic sub-model was defined in the Ansys Mechanical APDL. The mathematical description was based on the commonly employed equation, where the magnetic vector potential $A$ is applied.

$$
\nabla \times\left(\frac{1}{\mu} \nabla \times \mathbf{A}\right)+j \omega \sigma \mathbf{A}=\mathbf{J}_{\mathbf{s}},
$$

where $\mu$ and $\sigma$ are the magnetic permeability and conductivity of aluminium, respectively, $\omega$ is the angular frequency and $J_{s}$ is the current density source.

Based on the distribution of the magnetic vector potential $A$, the distribution of the magnetic induction $B$ (eqn (2)), the eddy current densities $J$ (eqn (3)) and the density of the electromagnetic force that acts on a liquid metal (eqn (4)) can be determined:

$$
\begin{gathered}
\mathbf{B}=\nabla \times \mathbf{A} \\
\mathbf{J}=-j \omega \sigma \mathbf{A}
\end{gathered}
$$

The information that was transferred to the fluid dynamics sub-model was the electromagnetic force $F_{\mathrm{EMAG}}$ and the power loss $q_{\mathrm{EMAG}}$ inside a melt. These values were obtained by the following equations:

$$
\mathbf{F}_{E M A G}=\frac{1}{2} \operatorname{Re}\left(\mathbf{J} \times \mathbf{B}^{*}\right)
$$




$$
q_{\text {EMAG }}=\frac{|\mathbf{J}|^{2}}{\sigma}
$$

The fluid dynamics sub-model was defined in Ansys Fluent. To predict the free surface shape, the volume fraction and momentum conservation equations had to be solved. These equations for the considered geometry are as follows:

$$
\begin{gathered}
\frac{\partial}{\partial t}\left(\alpha_{q} \rho_{q}\right)+\nabla\left(\alpha_{q} \rho_{q} \mathbf{v}\right)=0 \\
\frac{\partial}{\partial t}(\rho \mathbf{v})+\nabla(\rho \mathbf{v} \mathbf{v})=-\nabla p+\mu \nabla^{2} \mathbf{v}+\rho \mathbf{g}+\mathbf{F}_{E M A G}+\mathbf{F}_{s},
\end{gathered}
$$

where $t$ is the time, $\alpha_{q}$ is the volume fraction of the $q^{\text {th }}$ phase, $\rho_{q}$ is the density of the $q^{\text {th }}$ phase, $\mathbf{v}$ is the velocity vector, $p$ is the pressure, $\mu$ is the dynamic viscosity, $\mathbf{g}$ is the gravitational acceleration vector, $\mathbf{F}_{\mathrm{EMAG}}$ is the Lorentz Force, and $\mathbf{F}_{\mathrm{s}}$ is the surface tension force.

To simulate the impurities removal process, a conservation equation for chemical species was solved.

$$
\frac{\partial}{\partial t}\left(\rho Y_{i}\right)+\nabla \cdot\left(\rho \mathbf{v} Y_{i}\right)=-\nabla \cdot \mathbf{J}_{i},
$$

where $Y_{i}$ is the local mass fraction of each species, and $\mathbf{J}_{i}$ is the diffusion flux of species $i$.

A flow within an induction furnace crucible is strongly turbulent due to intensive electromagnetic mixing. Therefore, it was necessary to employ a turbulence model, i.e., k- $\varepsilon$.

The defined set of differential equations was completed by the material properties. The properties of the molten copper and lead alloy were as follows: density of $8000 \mathrm{~kg} / \mathrm{m}^{3}$, and

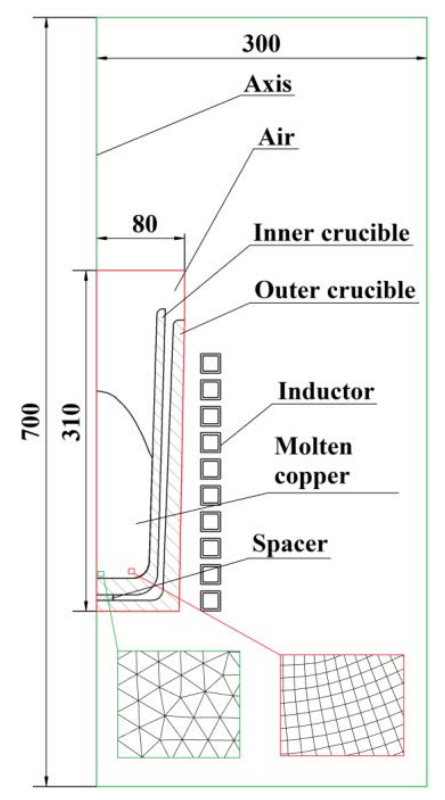

Figure 1: Simplified geometry of numerical domain for both electromagnetic (green line) and fluid dynamics (red line) sub-models [6]. 
dynamic viscosity of $0.00375 \mathrm{~Pa} \cdot \mathrm{s}$. To simplify the model, the air was examined as a gas with the constant material properties $\rho=1.225 \mathrm{~kg} / \mathrm{m}^{3}$ and $\mu=1.7894 \mathrm{e}-5 \mathrm{~Pa} \cdot \mathrm{s}$. The mass diffusivity was assumed to be a constant value of $2 \mathrm{e}-8 \mathrm{~m}^{2} / \mathrm{s}$. To model lead transport through the free surface, the total mass transport coefficient of $6 \mathrm{e}-5 \mathrm{~m} / \mathrm{s}$ was determined according to the experiment data [7].

\section{RESULTS AND DISCUSSION}

To examine the influence of different operating conditions of the induction furnace, the computations were performed for 5 variants. For every variant, a different inductor power was assumed. In Table 1, the applied values of the root mean square current and the frequency are listed. In Fig. 2, the molten metal free surface shape for every case is presented. The highest meniscus was observed for Variant 5, whereas the free surface of Variant 1 exhibited the flattest meniscus. An increase in the inductor power should directly correspond to an increase in the Lorentz force, which was induced in the charge. As a result, a higher meniscus was formed for a higher input power.

Once the meniscus was formed and stable, the purification process was analysed. The computations for the real time of $30 \mathrm{~min}$ were performed. The initial value of the lead mass fraction in the charge was $2 \%$, which was equally distributed. The values of the averaged lead mass fraction and a free surface area after $30 \mathrm{~min}$ of the process is listed in Table 2 . The smallest area and the highest averaged lead mass fraction were observed for Variant 1, which is characterised by the smallest input power. With an increase in inductor power, the meniscus area increased, and the averaged lead mass fraction decreased. For Variant 5, more

Table 1: Root mean square current and frequency for five different operating conditions.

\begin{tabular}{|l|l|l|}
\hline Case & $\begin{array}{l}\text { Root mean square } \\
\text { current }\end{array}$ & Frequency \\
\cline { 2 - 3 } & $\mathrm{A}$ & $\mathrm{Hz}$ \\
\hline Variant 1 & 1237 & 2940 \\
\hline Variant 2 & 1697 & 2830 \\
\hline Variant 3 & 2121 & 2800 \\
\hline Variant 4 & 2449 & 2800 \\
\hline Variant 5 & 2738 & 2800 \\
\hline
\end{tabular}
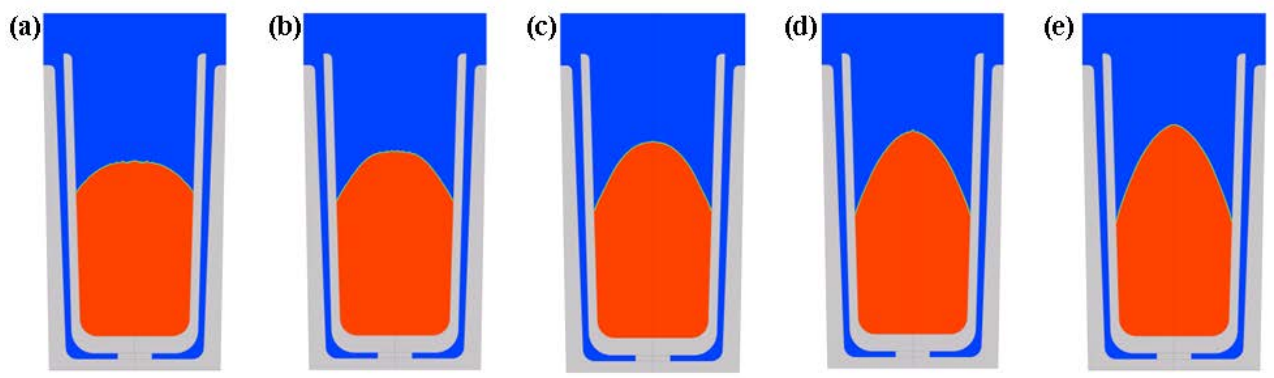

Figure 2: Molten metal (red) and air (blue) distribution for every computed case (Variant $1-(a)$, Variant $2-(b)$, Variant $3-(c)$, Variant $4-(d)$, Variant $5-(e))$. 
Table 2: Free surface area and lead averaged mass fraction after 30 min of process.

\begin{tabular}{|l|l|l|}
\hline Case & Free surface area & $\begin{array}{l}\text { Lead averaged mass } \\
\text { fraction after 30 min }\end{array}$ \\
\cline { 2 - 3 } & $\mathrm{m}^{2}$ & $\%$ \\
\hline Variant 1 & 0.0111 & 1.44 \\
\hline Variant 2 & 0.0136 & 1.33 \\
\hline Variant 3 & 0.0162 & 1.17 \\
\hline Variant 4 & 0.0182 & 1.06 \\
\hline Variant 5 & 0.0203 & 0.92 \\
\hline
\end{tabular}

Lead Mass Fraction
\begin{tabular}{|l}
$1.063 \mathrm{e}-002$ \\
$1.062 \mathrm{e}-002$ \\
$1.061 \mathrm{e}-002$ \\
$1.060 \mathrm{e}-002$ \\
$1.059 \mathrm{e}-002$ \\
$1.058 \mathrm{e}-002$ \\
$1.057 \mathrm{e}-002$ \\
$1.056 \mathrm{e}-002$ \\
$1.055 \mathrm{e}-002$ \\
$1.054 \mathrm{e}-002$ \\
$1.053 \mathrm{e}-002$
\end{tabular}

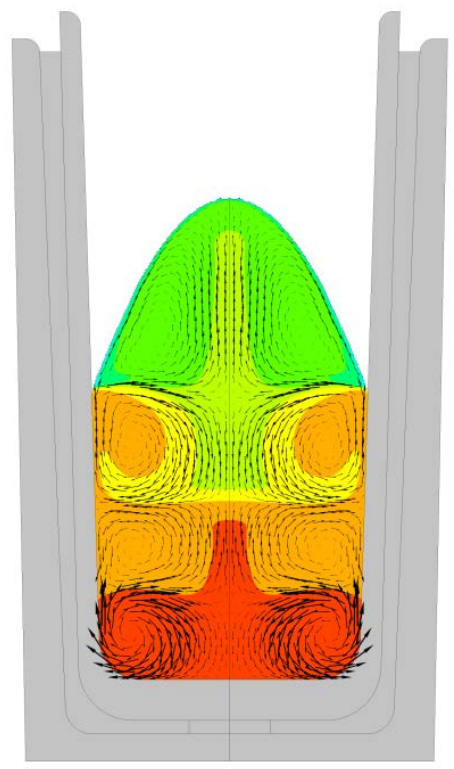

Figure 3: Lead mass fraction and velocity vectors for Variant 3 after 30 min of the process.

than $50 \%$ of the initial mass of lead was removed from the charge. A distribution of the lead mass fraction and the velocity vectors was presented in Fig. 3. The mass fraction of lead is uniformly distributed, which indicates a difference between the lowest value and the highest value of approximately $0.01 \%$. The highest concentration of lead was observed for the lower region of the charge. An area near the free surface was characterised by the lead lower mass fraction. The lead distribution is strongly combined with the vortices that were formed within the molten metal.

\section{SUMMARY}

The objective of this study was a numerical model development of impurities removal process within an induction furnace. The purification process of copper and lead alloy was examined for five inductor powers. The influence of induction furnace power is crucial for meniscus formation. For a high input power, the intensity of the purification process is high 
because the area of free surface directly corresponds to the mass transport through the interface.

\section{ACKNOWLEDGEMENT}

Financial assistance was provided by grant no. 2014/13/B/ST8/02364, which was funded by the National Science Centre in Poland.

\section{REFERENCES}

[1] Spitans, S., Jakovics, A., Baake, E. \& Nacke, B., Numerical modelling of free surface dynamics of conductive melt in the induction furnace. Magnetohydrodynamics, 46, pp. 317-328, 2010.

[2] Song, J.H., Min, B.T., Kim, J.H., Kim, H.W., Hong, S.W. \& Chung, S.H., An electromagnetic and thermal analysis of a cold crucible melting. International Communications in Heat and Mass Transfer, 32, pp. 1325-1336, 2005.

[3] Liu, T. et al., Purification of metallurgical silicon through directional solidification in a large cold crucible. Journal of Crystal Growth, 355, pp. 145-150, 2012.

[4] Buliński, P. et al., Effect of turbulence modeling in numerical analysis of melting process in an induction furnace. Archives of Metallurgy and Materials, 60, pp. 15751579, 2015.

[5] Buliński, P., Smołka, J., Golak, S. \& Przyłucki, R., Coupled numerical model of metal melting in an induction furnace: sensitivity analysis and validation of model. Przeglad elektrotechniczny, 92(3), pp. 49-52, (2016).

[6] Buliński, P. et al., Numerical and experimental investigation of heat transfer process in electromagnetically driven flow within a vacuum induction furnace. Applied Thermal Engineering.

[7] Blacha, L., Elimination of lead and antimony from copper and its alloys in the vacuum refining process. Scientific Notes of Silesian University of Technology, pp. 56-59, 2001. (in Polish). 